\title{
Peculiar microwave quasi-periodic pulsations with zigzag pattern in a CME-related Flare on 2005-01-15
}

\author{
Baolin Tan ${ }^{1}$ \\ ${ }^{1}$ Key Laboratory of Solar Activity, National Astronomical Observatories of the Chinese \\ Academy of Sciences, Beijing 100012, China. \\ email: bltan@nao.cas.cn
}

\begin{abstract}
A microwave quasi-periodic pulsation with zigzag pattern (Z-QPP) in a solar flare on 2005-01-15 is observed by the Chinese Solar Broadband Spectrometer in Huairou (SBRS/Huairou) at 1.10-1.34 GHz. The zigzag pulsation occurred just in the early rising phase of the flare with weakly right-handed circular polarization. Its period is only several decades millisecond. Particularly, before and after the pulsation, there are many spectral fine structures, such as zebra patterns, fibers, and millisecond spikes. The microwave Z-QPP can provide some kinematic information of the source region in the early rising phase of the flare, and the source width changes from $\sim 1000 \mathrm{~km}$ to $3300 \mathrm{~km}$, even if we have no imaging observations. The abundant spectral fine structures possibly reflect the dynamic features of non-thermal particles.
\end{abstract}

Keywords. solar flare, microwave bursts, fine structures

Solar microwave spectral fine structures are the most important and interesting phenomena, which can provide many intrinsic features of solar eruptions. This work reports first time a peculiar fine structure: a microwave quasi-periodic pulsation (QPP) with zigzag pattern (abbreviated as Z-QPP, hereafter) in a flare on 2005-01-15.

The flare is an M8.6 class long-duration event accompanying with a powerful CME in active region AR10720 with location of N16E04, very close to the center of solar disk. It
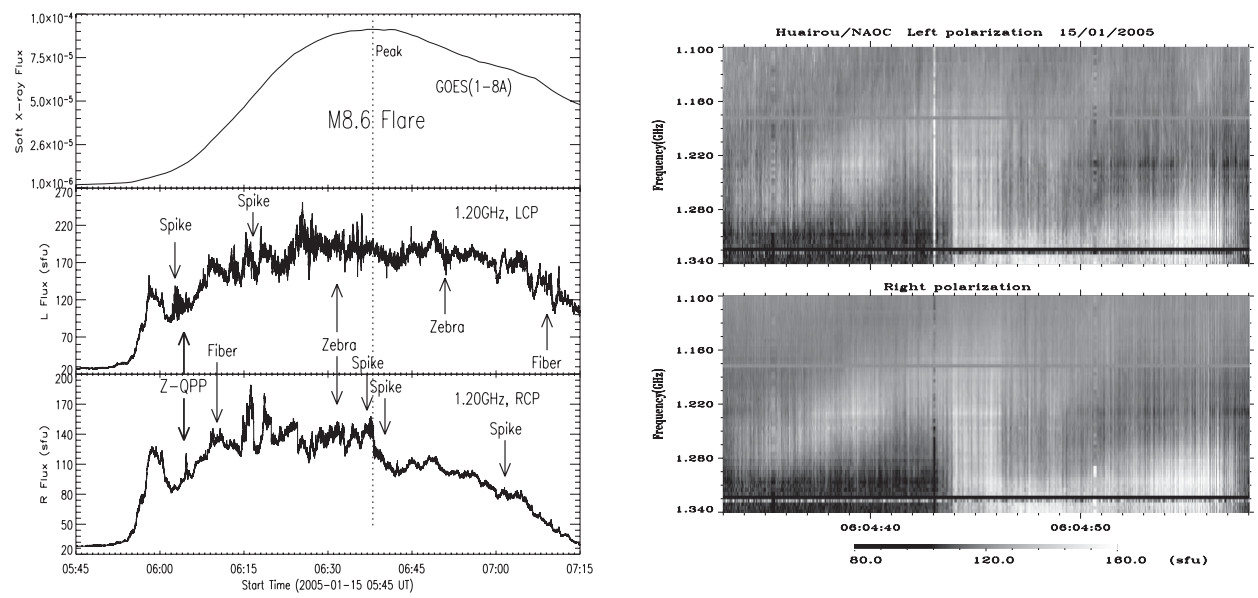

Figure 1. Left panels are the profiles of the M8.6 long-duration flare on 2005-1-15. Soft X-ray intensity observed by GOES (upper), microwave emission of left-handed circular polarization at $1.20 \mathrm{GHz}$ (middle), and the microwave emission of right-handed circular polarization at 1.20 $\mathrm{GHz}$ (bottom). Right panels are the spectrogram of microwave quasi-periodic pulsation with zigzag pattern (Z-QPP) in the early rising phase of the flare. 
is observed by the Chinese Solar Broadband Spectrometer in Huairou (SBRS/Huairou) at frequency of $1.10-1.34 \mathrm{GHz}(4.0 \mathrm{MHz}$ resolution, $1.25 \mathrm{~ms}$ cadence). The left panels of Figure 1 show that the flare lasts from 05:54 UT to 07:17 UT, for about 83 minutes (Cheng et al. 2010). The Z-QPP occurred just in the early rising phase of the flare. The right panels present the Z-QPP with left- and right-handed circular polarization spectrogram. The Z-QPP is weakly right-handed circular polarization, its period is only several decades of milliseconds, which belongs to very short period pulsation. We may partition the Z-QPP into three paragraphs. The first paragraph starts at 06:04:32 UT, ends at 06:04:43 UT, the global frequency drifting rate is about $-16.5 \mathrm{MHz} / \mathrm{s}$, the pulse frequency drifting rate is $2.30-9.60 \mathrm{GHz} / \mathrm{s}$, the period is $40-85 \mathrm{~ms}$ with averaged value of $60 \mathrm{~ms}$. The bandwidth increases slowly from $60 \mathrm{MHz}$ to $160 \mathrm{MHz}$. The second paragraph starts at 06:04:43 UT and ends at 06:04:47 UT, its global frequency drifting rate is about 0 and the pulse frequency drifting rate is $9.60-14.40 \mathrm{GHz} / \mathrm{s}$, the period is $48-106 \mathrm{~ms}$, and the bandwidth 140-200 MHz, few variations. The third paragraph starts at 06:04:47 UT and ends at 06:04:47 UT, its global frequency drifting rate is about $-5.6 \mathrm{MHz} / \mathrm{s}$ and the pulse frequency drifting rate is $6.7-20 \mathrm{GHz} / \mathrm{s}$, the period is $38-87 \mathrm{~ms}$, and the bandwidth increases slowly from 60 to $140 \mathrm{MHz}$, and possibly beyond frequency domain $(1.34 \mathrm{GHz})$.

The frequency distance between the two adjacent strips of the concomitant zebra pattern is about $80 \mathrm{MHz}$, which implies that the magnetic field in the source region is about 71.5 Gs from DPR model. The weakly polarization and strongly emission intensity imply that plasma emission is most possibly the emission mechanism. The Z-QPP may reflect the variations of the physical conditions and the dynamic processes in source region (Aschwanden \& Benz, 1986; Tan, 2008). The velocities of source motion or energetic particles $(v)$, and the width of source region $(\mathrm{L})$ can be estimated:

$$
v=2 \frac{d f}{f d t} H_{n}, \quad L=2 \frac{\triangle f}{f} H_{n} .
$$

$H_{n}$ is the scale length of plasma density in the background for the source motion and in the source region for the energetic particles.

The above estimation of the Z-QPP indicates the source region moves in a speed from 275-93 km/s upwards, the source width expands from $1000 \mathrm{~km}$ to $3300 \mathrm{~km}$, and the associated speeds of energetic particles is about $0.130 .53 c, 0.53-0.8 c$, and $0.36-0.9 c$ in the three paragraphs, respectively. Here, $c$ is the light speed. Before and after the Z-QPP, there are many spectral fine structures, such as zebra patterns, fibers and millisecond spikes, etc. Some of them are marked in Figure 1. The abundant spectral fine structures reflect the dynamic features of the non-thermal particles (Huang \& Tan, 2012).

\section{Acknowledgements}

This work is supported by NSFC Grant No. 11273030, 10921303, MOST Grant No. 2011CB811401, and the National Major Scientific Equipment R\&D Project ZDYZ2009-3.

\section{References}

Aschwanden, M. J., Benz A. O. 1986, Astron Astrophys, 158, 102

Cheng, X., Ding, M. D., Guo, Y., et al. 2010, ApJ, 716, L68

Huang, J. \& Tan, B. L. 2012, ApJ, 745, 186

Qin, Z. H., Li, C. S., Fu, Q. J., et al. 1996, Sol. Phys., 163, 383

Tan, B. L. 2008, Sol. Phys., 253, 117 